\title{
Pricing American Perpetual Warrants by Linear Programming*
}

\begin{abstract}
A warrant is an option that entitles the holder to purchase shares of a common stock at some prespecified price during a specified interval. The problem of pricing a perpetual warrant (with no specified interval) of the American type (that can be exercised any time) is one of the earliest contingent claim pricing problems in mathematical economics. The problem was first solved by Samuelson and McKean in 1965 under the assumption of a geometric Brownian motion of the stock price process. It is a well-documented exercise in stochastic processes and continuous-time finance curricula. The present paper offers a solution to this time-honored problem from an optimization point of view using linear programming duality under a simple random walk assumption for the stock price process, thus enabling a classroom exposition of the problem in graduate courses on linear programming without assuming a background in stochastic processes.
\end{abstract}

Key words. pricing, perpetual warrant, American option, linear programming, duality, dynamic programming, harmonic functions, second-order difference equations

AMS subject classifications. 90C05, 90C39, 90C90, 31C05, 91B28

DOI. $10.1137 / 080728366$

I. Introduction. A warrant is an option that entitles the holder to buy shares of a common stock at some prespecified price (referred to as the "exercise price") during a specified interval. A perpetual warrant is a warrant that does not have an expiration date. An American perpetual warrant (which can also be called a perpetual American option) can be exercised at any time. The problem of pricing an American perpetual warrant is to determine the fair price that an investor should be willing to pay to acquire it. It is a fundamental problem in the fields of financial economics and mathematical finance. The explicit solution for the pricing of warrants was given by McKean (in the appendix of [12]) under the assumption of a geometric Brownian motion model for the stock price process, upon a question posed by Samuelson [12] in 1965. A textbook coverage of the solution is given in An Introduction to Stochastic Processes by Kao [10], in Example 7.1.3, which is in turn based on Karlin and Taylor

*Received by the editors June 24, 2008; accepted for publication (in revised form) July 24, 2009; published electronically November 6, 2009.

http://www.siam.org/journals/sirev/51-4/72836.html

$\dagger$ Department of Operations Research and Financial Engineering, Princeton University, Princeton, NJ 08544 (rvdb@princeton.edu).

${ }^{\ddagger}$ Department of Industrial Engineering, Bilkent University, 06800 Ankara, Turkey (mustafap@ bilkent.edu.tr). The research in this paper was done while this author was on sabbatical leave from Bilkent University at the Department of Operations and Financial Engineering, Princeton University, and was supported by a Fulbright Senior Grant and TÜBİTAK grant 107K250. 
[11]. Chen [3] gives a solution for finite-time warrants. A recent account of the subject along with developments after 1965 can be found in [13].

In this note, we offer a solution based on linear programming duality, after assuming that the stock price behaves as a simple random walk. The purpose is to offer yet another application where the well-developed machinery of linear programming can be brought to bear upon a time-honored problem of financial economics in a classroom setting which does not require a background in stochastic calculus and continuous-time finance. On the other hand, the paper can also serve researchers in optimization and mathematical programming seeking an introduction to the field of mathematical finance. Another interesting feature of the solution methodology is the connection to the theory of harmonic functions as treated in the excellent book by Dynkin and Yushkevich [6]. On several occasions, concepts familiar to the student of harmonic functions appear in simple forms in our elementary analysis where we try to refer the reader each time to an appropriate source.

Let $X_{n}$ denote the share price of the stock at a time $n$ periods into the future. Obviously, $X_{n}$ is a random variable, and the collection of these random variables over time is a stochastic process. Let us assume that $X_{n}$ is a random walk with absorption at 0 on the state space $E=\{j \Delta x: j=0,1,2, \ldots\}$, where $\Delta x$ is a fixed small positive real number. That is, if the current stock price is $x \in E$, then the price at the next period will be either $x+\Delta x$ or $x-\Delta x$ with probabilities $p$ and $q=1-p$, respectively. This model of stock price movements is close to reality in that stock prices usually move by small amounts over sufficiently short periods of time.

In order to figure out how to price the warrant, one must answer the question, what is the expected value, in today's dollars, of the warrant? Naturally, the seller of the warrant should assume that the buyer will employ an optimal strategy for exercising the warrant. If at some future date, the stock price $X_{n}$ is larger than the exercise price $S$, then the holder of the warrant can buy the stock for $S$ dollars and immediately sell it for $X_{n}$ dollars and realize a gain of $X_{n}-S$ dollars. Hence, the payoff at time $n$ of the warrant is $h\left(X_{n}\right)=X_{n}-S$. If the warrant holder exercises the option at some time $\tau$ (possibly random, but not clairvoyant), then the expected present value would be

$$
\mathbb{E}_{x} \alpha^{\tau} h\left(X_{\tau}\right)
$$

Here we have introduced a discount factor $\alpha$ (a number slightly less than one). This discount factor accounts for the fact that future dollars are worth less than present dollars. Specifically, $\alpha$ gives today's value of tomorrow's dollar. The optimal strategy is then determined by maximizing over all nonclairvoyant random times $\tau$ :

$$
v(x)=\max _{\tau} \mathbb{E}_{x} \alpha^{\tau} h\left(X_{\tau}\right)
$$

The function $v$ is called the value function. It tells both the buyer and the seller of the warrant everything they need to know. To the seller, the "fair" price for the warrant is $v(x)$ if the current stock price is $x$. Since zero payoff can be obtained with the strategy $\tau=+\infty$, the function $v$ is nonnegative.

In each period, the holder of the warrant observes the current price $x$ of the stock and decides either to exercise the warrant or to keep the warrant for one more period. The optimal choice between exercising the warrant and holding it for one more period depends on the relative magnitudes of the payoff $h(x)$ and the expected warrant value one time step ahead, i.e., $\alpha(p v(x+\Delta x)+q v(x-\Delta x))$. By "the principle of dynamic 
programming" ${ }^{1} v(x)$ must satisfy the equation

$$
v(x)=\max \{h(x), \alpha(p v(x+\Delta x)+q v(x-\Delta x))\}
$$

for all $x \in E \backslash\{0\}$, and the boundary condition $v(0)=0$. This equation characterizes the optimal strategy for the buyer of the warrant. The state space can be partitioned into two sets: the set of states $x$ where $v(x)>h(x)$ and those where $v(x)=h(x)$. The first set is called the "continuation region" and the second the "exercise region" [5]. The buyer should not exercise the option at times $n$ when $X_{n}$ is in the continuation region, that is, when $v\left(X_{n}\right)>h\left(X_{n}\right)$. In fact, the buyer should exercise the warrant as soon as $v\left(X_{n}\right)=h\left(X_{n}\right)$.

Based on the implicit equation above, it is shown in probability courses (see, e.g., [6, Chapter 3, pp. 106-107] or [4, Chapter 7, pp. 212-213]) that $v$ can be uniquely characterized as the smallest (in the $L^{1}$ sense) function that satisfies these inequalities:

$$
\begin{array}{ll}
v(x) \geq 0, & x \in E, \\
v(x) \geq h(x), & x \in E, \\
v(x) \geq \alpha(p v(x+\Delta x)+q v(x-\Delta x)), & x \in E \backslash\{0\} .
\end{array}
$$

Clearly, when the price of the stock price $x$ is less than $S$ there is no incentive to exercise the option - the warrant holder would lose money needlessly. Hence, we can redefine the warrant payoff function as $f(x)=(x-S)^{+}$and replace the first two set of inequalities above by the single set of inequalities $v(x) \geq f(x)$ for all $x \in E$. Recalling the discreteness of our model's state space, we can formulate an infinite-dimensional linear programming problem

$$
\begin{array}{lll}
\operatorname{minimize} & \sum_{j=0}^{\infty} v_{j} & \\
\text { subject to } & v_{j} \geq f_{j}, & j=0,1,2, \ldots, \\
& v_{j} \geq \alpha\left(p v_{j+1}+q v_{j-1}\right), & j=1,2, \ldots,
\end{array}
$$

where $x_{j}=j \Delta x, v_{j}=v\left(x_{j}\right)$, and $f_{j}=f\left(x_{j}\right)$.

Let $v_{j}^{*}, j=0,1, \ldots$, denote the optimal solution to this linear programming problem. Figure 1 gives an illustration of $v^{*}(x)$ and $f(x)$ corresponding to the case where $\alpha=0.999, p=0.5, \Delta x=0.1$, and $S=9$. From the original real-world description of the problem and Figure 1, it appears that the optimal strategy for exercising the warrant would be not to exercise when the stock price is below some threshold and then exercise as soon as the stock price hits the threshold value. In other words, it seems reasonable to conjecture that under some mild assumptions which we shall list below

\footnotetext{
${ }^{1}$ The principle of dynamic programming, a.k.a. the principle of optimality, states, "An optimal policy has the property that whatever the initial state and the initial decision are, the remaining decision must constitute an optimal policy with regard to the state resulting from the initial decision." See Bellman and Dreyfus [1].
} 


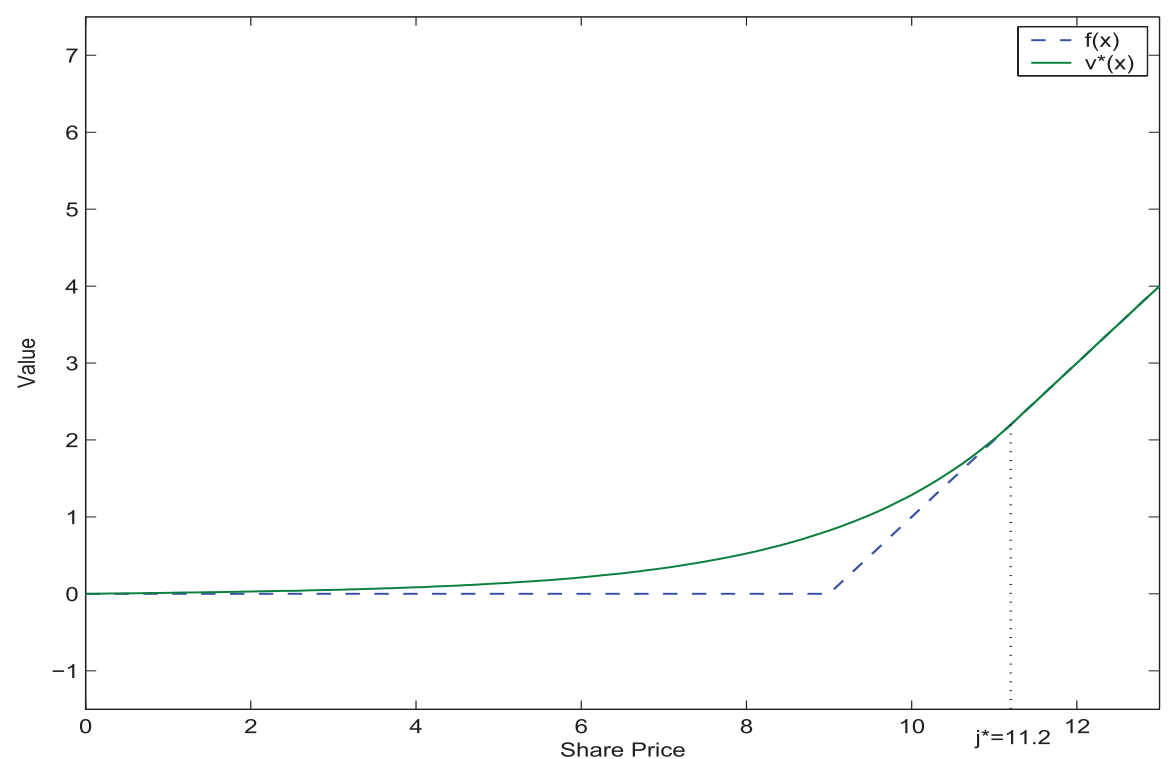

Fig. I Plot of $v^{*}(x)$ and $f(x)$ corresponding to the case where $\alpha=0.999, p=0.50, \Delta x=0.1$, and $S=9$. In this case, $j^{*}=112$ and therefore $x_{j^{*}}=11.2$.

there exists an optimal solution with the structure

$$
\begin{aligned}
& v_{0}^{*}=f_{0}, \\
& v_{j}^{*}=\alpha\left(p v_{j+1}^{*}+q v_{j-1}^{*}\right)>f_{j}, \quad 0<j<j^{*}, \\
& v_{j}^{*}=f_{j} \quad>\alpha\left(p v_{j+1}^{*}+q v_{j-1}^{*}\right), \quad j^{*} \leq j,
\end{aligned}
$$

for some appropriately chosen $j^{*}$.

Our guess is indeed correct. Indeed, if we let

$$
\xi_{-}=\frac{-1-\sqrt{1-4 \alpha^{2} p q}}{-2 \alpha p}, \quad \xi_{+}=\frac{-1+\sqrt{1-4 \alpha^{2} p q}}{-2 \alpha p}
$$

and assume that

1. $\alpha p \leq 1 / 2$ and $\alpha q \leq 1 / 2$,

2. $S=j_{S} \Delta x$ for some integer $j_{S}$, and

3. $f_{j_{S}+1}\left(\xi_{+}^{j_{S}}-\xi_{-}^{j_{S}}\right) /\left(\xi_{+}^{j_{S}+1}-\xi_{-}^{j_{S}+1}\right)>f_{j_{S}}$,

we can then give a closed-form formula for $j^{*}$ and the optimal values of $v_{j}$ :

$$
\begin{gathered}
j^{*}=\max \left\{k: f_{k} \frac{\xi_{+}^{k-1}-\xi_{-}^{k-1}}{\xi_{+}^{k}-\xi_{-}^{k}}>f_{k-1}\right\}, \\
v_{j}= \begin{cases}0, & j=0, \\
f_{j^{*}} \frac{\xi_{+}^{j}-\xi_{-}^{j}}{\xi_{+}^{j *}-\xi_{-}^{j^{*}}} & 0<j<j^{*}, \\
f_{j}, & j^{*} \leq j .\end{cases}
\end{gathered}
$$

In the problem instance of Figure 1, the optimal choice of $j^{*}$ is 112. In Figures 2 and 3 , we show the consequences of choosing $j^{*}$ too small or too large for the same problem 


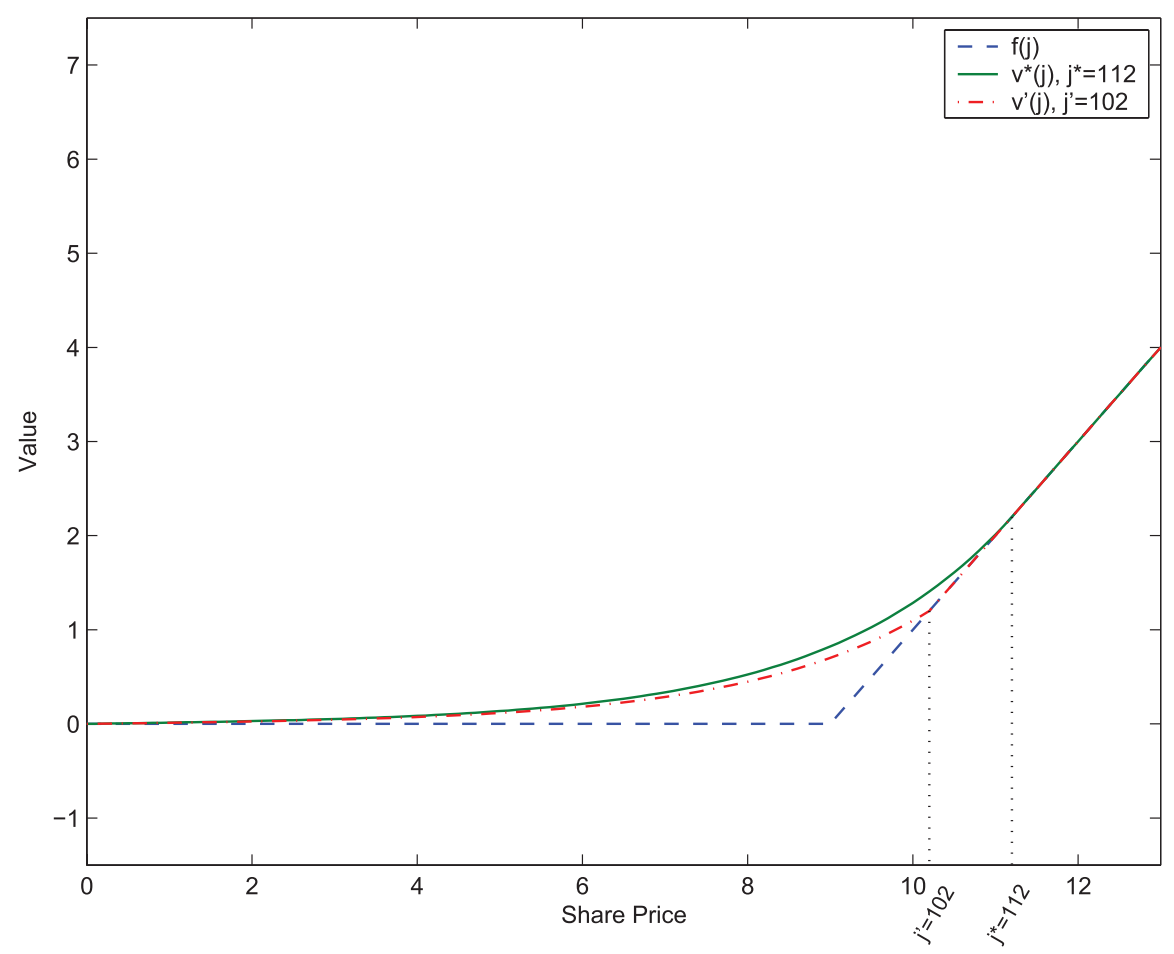

Fig. 2 Plot of $v^{\prime}(j), v^{*}(j)$, and $f(j)$ when $j^{\prime}=102$ and $j^{*}=112$.

instance. Suppose we were unable to correctly determine the optimal exercise point $j^{*}$. That is, we assume that $j^{\prime}$ determines the boundary continuation and exercise regions but that $j^{\prime} \neq j^{*}$. In this case, the expected payoff $v^{\prime}(j)$ is given by

$$
v^{\prime}(j)= \begin{cases}0, & j=0, \\ f_{j^{\prime}} \frac{\xi_{+}^{j}-\xi_{-}^{j}}{\xi_{+}^{j^{\prime}}-\xi_{-}^{j^{\prime}}}, & 0<j<j^{\prime} \\ f_{j}, & j^{\prime} \leq j .\end{cases}
$$

If we set $j^{\prime}=102$, then we obtain the plot shown in Figure 2. When we set $j^{\prime}=122$ the resulting plot is as shown in Figure 3. In both cases the $v$-curves are below the optimal $v$-curve obtained with the optimal choice $j^{*}$. In the first case, the $v$ values violate some of the inequalities $v_{j} \geq \alpha\left(p v_{j+1}+q v_{j-1}\right)$, and in the second case, some of the inequalities $v_{j} \geq f_{j}$ are violated. In the case of Figure 2, the holder of the option erroneously underestimates the value of the warrant. This underestimation leads to an exercise of the warrant at a share price where it is in fact still advantageous to defer exercise. In the case of Figure 3, the incorrect valuation induces the holder to defer exercise at stock prices where it is in fact optimal to exercise, thereby foregoing income that could earn risk-free interest during the delay.

A function $v^{*}$ defined on $0<j<j^{*}$ satisfying $v_{j}^{*}=\alpha\left(p v_{j+1}^{*}+q v_{j-1}^{*}\right)$ is known as an $\alpha$-harmonic function. A function $v^{*}$ defined for all $j \geq j^{*}$ and satisfying $v_{j}^{*}>\alpha\left(p v_{j+1}^{*}+q v_{j-1}^{*}\right)$ is called an $\alpha$-superharmonic function. For such definitions, the reader is directed to the opening chapter of [6]. 


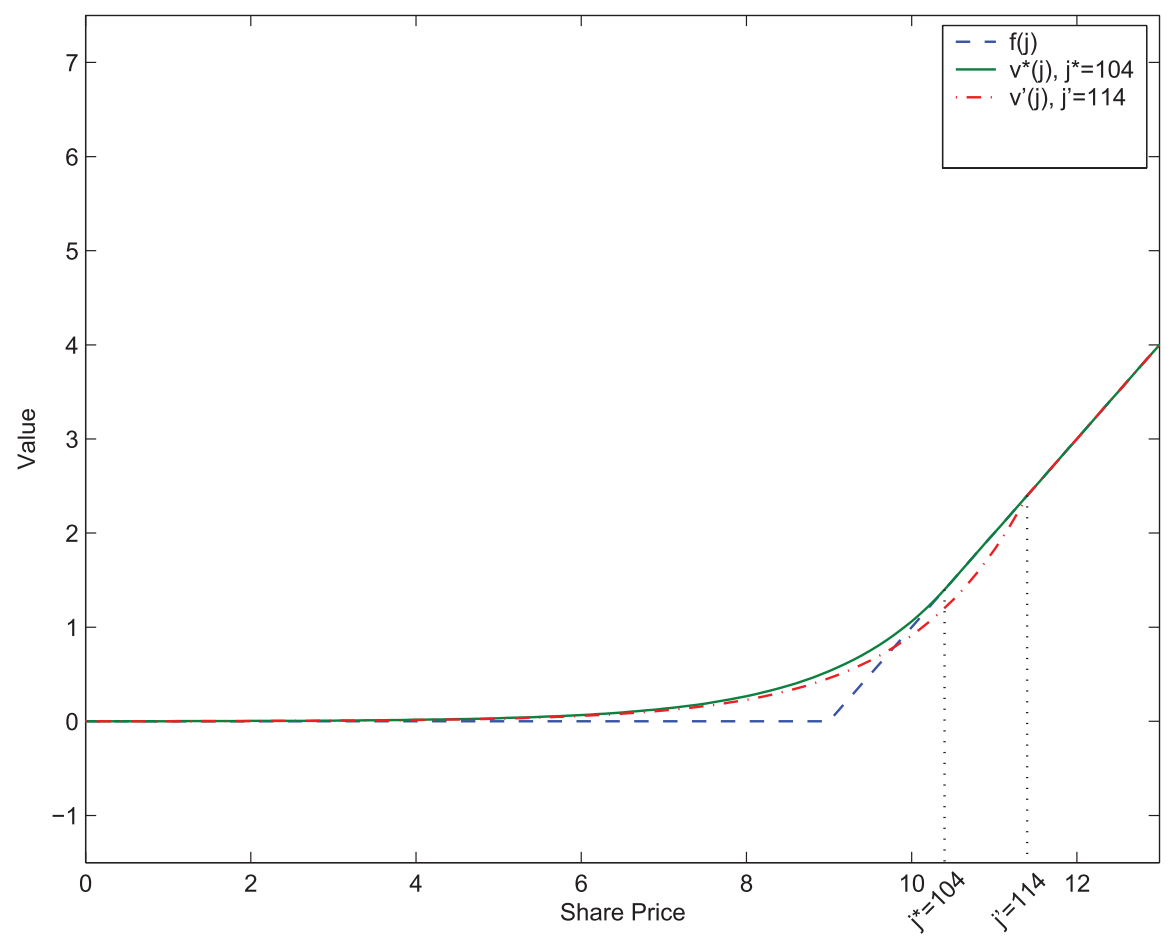

Fig. 3 Plot of $v^{\prime}(j), v^{*}(j)$, and $f(j)$ when $j^{\prime}=122$ and $j^{*}=112$.

In the rest of this paper, we shall give a step-by-step derivation of the closed-form formulae (1.1)-(1.2) and demonstrate the correctness of our guess at the solution using linear programming duality and elementary techniques for the solution of second-order difference (differential) equations. For a review of the fundamental results of linear programming, our desktop reference is the textbook [14]. For a coverage of difference equations that is suitable for our purposes, the reader can consult [9].

The plan is as follows. We first lay out the primal and dual linear programming problems, and use the complementary slackness property of optimal solutions to linear programs to simplify the claimed form of the equations leading to an optimal solution into a pair of second-order difference equations. We compute solutions to these secondorder difference equations and show how these solutions satisfy various inequalities defining the primal and dual problems. Along the way, we discover the few mild assumptions that need to be made for our guess to be correct.

2. Primal Problem. Let us restate the primal linear programming problem:

$$
\begin{array}{lll}
\operatorname{minimize} & \sum_{j=0}^{\infty} v_{j} & \\
\text { subject to } & v_{j} \geq f_{j}, & j \geq 0, \\
& v_{j} \geq \alpha\left(p v_{j+1}+q v_{j-1}\right), & j \geq 1 .
\end{array}
$$

Copyright (c) by SIAM. Unauthorized reproduction of this article is prohibited. 
3. Dual Problem. Duality theory plays a fundamental role in linear programming. The basic idea is that associated with every linear programming problem, there is another linear programming problem, which is called the dual to the first problem (and therefore the first problem is called the primal). The two most important results are the so-called weak and strong duality theorems. The weak duality theorem says that

for any set of values for the dual problem that satisfy the constraints of the dual problem, the value of the dual objective function evaluated with those values provides a (lower) bound on the optimal value to the primal problem.

The strong duality theorem goes further and says that

the optimal value of the dual objective function equals the optimal value of the primal objective function.

One of the corollaries of the strong duality theorem gives us a so-called certificate of optimality. This corollary says that

if one can exhibit values for the primal problem that satisfy the primal constraints and values for the dual problem that satisfy the dual constraints and if these primal and dual values satisfy an easy-to-check property called complementarity, then the primal values are optimal for the primal problem (and the dual values are optimal for the dual problem).

This corollary is extremely useful in that it reduces an optimization problem to a problem of simply checking that a few conditions are satisfied. This is how we shall use duality in what follows. See Chapter 5 of [2] or Chapter 5 of [14] for details on duality theory.

The dual problem is obtained by the following procedure. First, a Lagrangian function is constructed using a nonnegative Lagrange (or dual) multiplier value for each constraint. The Lagrangian function is then minimized with respect to the primal variables. The resulting function value constitutes a lower bound to the optimal value of the primal problem. Then maximizing this bound over all permissible choices of Lagrange multipliers gives the dual problem.

In our case the dual problem is

$$
\begin{array}{lll}
\text { maximize } & \sum_{j=0}^{\infty} f_{j} y_{j} & \\
\text { subject to } & y_{0}-\alpha q z_{1}=1, & \\
& y_{1}+z_{1}-\alpha q z_{2}=1, & \\
& y_{j}-\alpha p z_{j-1}+z_{j}-\alpha q z_{j+1}=1, & j \geq 2, \\
& y_{j} \geq 0, & j \geq 0, \\
& z_{j} \geq 0, & j \geq 1 .
\end{array}
$$

To see that this is correct, we shall derive the dual problem using the aforementioned procedure. Using nonnegative multipliers $y_{j}, j=0,1, \ldots$, for the first set of inequalities in the primal problem, and nonnegative multipliers $z_{j}, j=1,2, \ldots$, for the second set of inequalities, we obtain the Lagrangian function

$$
L(\mathbf{v}, \mathbf{y}, \mathbf{z})=\sum_{j=0}^{\infty} v_{j}+\sum_{j=0}^{\infty} y_{j}\left(f_{j}-v_{j}\right)+\sum_{j=1}^{\infty} z_{j}\left(\alpha p v_{j+1}+\alpha q v_{j-1}-v_{j}\right),
$$


where we denote the sequences $v_{j}, y_{j}$, and $z_{j}$ by $\mathbf{v}, \mathbf{y}$, and $\mathbf{z}$, respectively. To obtain the dual function, $g(\mathbf{y}, \mathbf{z})$ say, we have to minimize $L(\mathbf{v}, \mathbf{y}, \mathbf{z})$ over the unrestricted variables $v_{j}$; i.e., we have

$$
g(\mathbf{y}, \mathbf{z})=\min _{\mathbf{v}} L(\mathbf{v}, \mathbf{y}, \mathbf{z}) .
$$

We can rewrite this minimization problem as

$$
g(\mathbf{y}, \mathbf{z})=\sum_{j=0}^{\infty} f_{j} y_{j}+\min _{v}\left\{\sum_{j=0}^{\infty} y_{j}\left(f_{j}-v_{j}\right)+\sum_{j=1}^{\infty} z_{j}\left(\alpha p v_{j+1}+\alpha q v_{j-1}-v_{j}\right)\right\} .
$$

The minimization over each $v_{j}$ can be performed separately. For $v_{0}$ we have

$$
\min _{v_{0}} v_{0}\left(1-y_{0}+\alpha q z_{1}\right) .
$$

Since $v_{0}$ is unrestricted, we need to make the term in parentheses zero to obtain a finite minimum (equal to zero), which gives the first equation of the dual problem. Similarly, for $v_{1}$ we have

$$
\min _{v_{1}} v_{1}\left(1-y_{1}-z_{1}+\alpha q z_{2}\right)
$$

which yields the second equation of the dual problem. Finally, for $v_{j}, j \geq 2$, we perform the minimizations again separately to obtain the third set of equations in the dual problem.

4. Statement of Claim. Let $v_{j}$ denote the optimal primal solution and $y_{j}$ and $z_{j}$ the optimal dual solution (i.e., we are dropping the usual "stars" that denote optimality). Suppose, as claimed, that there exists a $j^{*}$ such that

$$
\begin{aligned}
& v_{0}=f_{0}, \\
& v_{j}=\alpha\left(p v_{j+1}+q v_{j-1}\right)>f_{j} \quad \text { for } 0<j<j^{*}, \\
& v_{j}=f_{j} \quad>\alpha\left(p v_{j+1}+q v_{j-1}\right) \quad \text { for } j^{*} \leq j .
\end{aligned}
$$

Now we are interested in solving the equations

$$
\begin{aligned}
& v_{0}=f_{0}, \\
& v_{j}=\alpha\left(p v_{j+1}+q v_{j-1}\right) \quad \text { for } 0<j<j^{*}, \\
& v_{j}=f_{j} \quad \text { for } j^{*} \leq j .
\end{aligned}
$$

5. Invoke Complementarity. Another useful property in linear programming is the complementarity theorem; see, e.g., [14, pp. 67-68]. In our particular case, it states that any optimal solution $v_{j}$ to the primal problem and any optimal solutions $y_{j}$ and $z_{j}$ to the dual problem satisfy

$$
\begin{aligned}
z_{j}\left(\alpha p v_{j+1}+\alpha q v_{j-1}-v_{j}\right) & =0, & & j \geq 1, \\
y_{j}\left(f_{j}-v_{j}\right) & =0, & & j \geq 0 .
\end{aligned}
$$

Copyright $\odot$ by SIAM. Unauthorized reproduction of this article is prohibited. 
Hence, complementarity equations (5.1) and (5.2) imply that

$$
\begin{array}{ll}
z_{j}=0, & j \geq j^{*}, \\
y_{j}=0, & 0<j<j^{*} .
\end{array}
$$

Since any optimal solution to the dual problem is also feasible (i.e., it satisfies all the equations of the dual problem) using (5.3), we have

$$
\begin{aligned}
y_{j^{*}}-\alpha p z_{j^{*}-1} & =1, \\
y_{j} & =1, \quad j>j^{*} .
\end{aligned}
$$

Similarly, using (5.4) we obtain

$$
\begin{aligned}
z_{1}-\alpha q z_{2} & =1, \\
-\alpha p z_{j-1}+z_{j}-\alpha q z_{j+1} & =1, \quad 1<j<j^{*} .
\end{aligned}
$$

6. Second-Order Difference Equations. The problem of solving (4.1)-(4.3) from the primal problem and the equations defining the dual problem has therefore been reduced to a pair of second-order difference equations with Dirichlet boundary conditions (i.e., boundary conditions that involve a known function) [9]. The first difference equation (for the primal) is

$$
\begin{aligned}
v_{j}-\alpha\left(p v_{j+1}+q v_{j-1}\right) & =0, \quad 0<j<j^{*}, \\
v_{0} & =0, \\
v_{j^{*}} & =f_{j^{*}},
\end{aligned}
$$

and the second one (for the dual) is

$$
\begin{aligned}
z_{j}-\alpha\left(p z_{j-1}+q z_{j+1}\right) & =1, \quad 0<j<j^{*}, \\
z_{0} & =0 \\
z_{j^{*}} & =0 .
\end{aligned}
$$

Note that in (6.2) we used the fact that $f_{0}=0$, and in (6.4) we have added a new variable, $z_{0}$, which is just fixed to zero (by (6.5)). In this way we consolidate the difference equation for $z_{j}$ to a more elegant form.

7. Solve the Difference Equations. First, we solve (6.1) for $v_{j}$. Suppose that $v_{j}=\xi^{j}$ for some positive real number $\xi$. Substituting into the difference equation, we get $\xi^{j}-\alpha\left(p \xi^{j+1}+q \xi^{j-1}\right)=0$. Dividing by $\xi^{j-1}$, we get a quadratic equation $-\alpha p \xi^{2}+\xi-\alpha q=0$ with the two roots

$$
\xi_{-}=\frac{-1-\sqrt{1-4 \alpha^{2} p q}}{-2 \alpha p}, \quad \xi_{+}=\frac{-1+\sqrt{1-4 \alpha^{2} p q}}{-2 \alpha p},
$$

where we used $\xi_{-}$to denote the larger root, which is greater than one, and $\xi_{+}$for the smaller root, which is strictly between zero and one (these properties hold if and only if $\alpha<1)$. The general solution to the difference equation is therefore $v_{j}=c_{+} \xi_{+}^{j}+c_{-} \xi_{-}^{j}$. Using the first boundary condition (6.2) and the second boundary condition (6.3), we obtain

$$
v_{j}=f_{j^{*}} \frac{\xi_{+}^{j}-\xi_{-}^{j}}{\xi_{+}^{j^{*}}-\xi_{-}^{j^{*}}}, \quad 0<j<j^{*}
$$

Copyright $@$ by SIAM. Unauthorized reproduction of this article is prohibited. 
Now, we solve for $z_{j}$ in (6.4). For a particular solution, we choose $z_{j} \equiv c$. Substituting into the difference equation, we get $c=1 /(1-\alpha)$. The general solution, which is the sum of the particular and the homogeneous solutions (the homogeneous equation is identical to (6.1) except for the interchange of $p$ and $q$ ), is given by $z_{j}=\frac{1}{1-\alpha}+c_{+} \zeta_{+}^{j}+c_{-} \zeta_{-}^{j}$, where

$$
\begin{aligned}
& \zeta_{+}=1 / \xi_{-}=\frac{-1+\sqrt{1-4 \alpha^{2} p q}}{-2 \alpha q}, \\
& \zeta_{-}=1 / \xi_{+}=\frac{-1-\sqrt{1-4 \alpha^{2} p q}}{-2 \alpha q} .
\end{aligned}
$$

Using the boundary conditions to eliminate the two undetermined constants, we get

$$
z_{j}=\left(1-\frac{\zeta_{-}^{j^{*}}-1}{\zeta_{-}^{j^{*}}-\zeta_{+}^{j^{*}}} \zeta_{+}^{j}-\frac{\zeta_{+}^{j^{*}}-1}{\zeta_{+}^{j^{*}}-\zeta_{-}^{j^{*}}} \zeta_{-}^{j}\right) /(1-\alpha), \quad 0<j<j^{*} .
$$

To summarize, we have

$$
\begin{aligned}
& v_{j}= \begin{cases}0, & j=0, \\
f_{j^{*}} \frac{\xi_{+}^{j}-\xi_{-}^{j}}{\xi_{+}^{j^{*}}-\xi_{-}^{j^{*}}}, & 0<j<j^{*}, \\
f_{j}, & j^{*} \leq j,\end{cases} \\
& z_{j}= \begin{cases}\left(1-\frac{\zeta_{-}^{j^{*}}-1}{\zeta_{-}^{j^{*}}-\zeta_{+}^{j^{*}}} \zeta_{+}^{j}-\frac{\zeta_{+}^{j^{*}}-1}{\zeta_{+}^{j^{*}}-\zeta_{-}^{j^{*}}} \zeta_{-}^{j}\right) /(1-\alpha), & 0<j<j^{*}, \\
0, & j=0,\end{cases} \\
& y_{j}= \begin{cases}1+\alpha q z_{1}, & 0<j<j^{*}, \\
0, & j=j^{*}, \\
1+\alpha p z_{j^{*}-1}, & j^{*}<j . \\
1, & \end{cases}
\end{aligned}
$$

In Figure 4 we illustrate the behavior of optimal dual variables $y$ and $z$ for the sample problem illustrated by Figure 1.

8. Check the Inequalities. All that remains is to show that the various inequalities are satisfied:

$$
\begin{array}{lr}
y_{j} \geq 0, & j \geq 0, \\
z_{j} \geq 0, & j \geq 1, \\
v_{j} \geq f_{j}, & j \geq 0, \\
v_{j} & \geq \alpha\left(p v_{j+1}+q v_{j-1}\right),
\end{array}
$$

8.I. Inequalities (8.2). Inequalities (8.2) follow trivially for $j \geq j^{*}$ from the formula given above for $z_{j}$. To check them for $j<j^{*}$, we do a proof by contradiction. So, suppose that $z_{j}<0$ for some $0<j<j^{*}$. Then there exists a $k$ at which $z_{k}$ is negative and a local minimum:

$$
z_{k} \leq z_{k-1} \quad \text { and } \quad z_{k} \leq z_{k+1}
$$



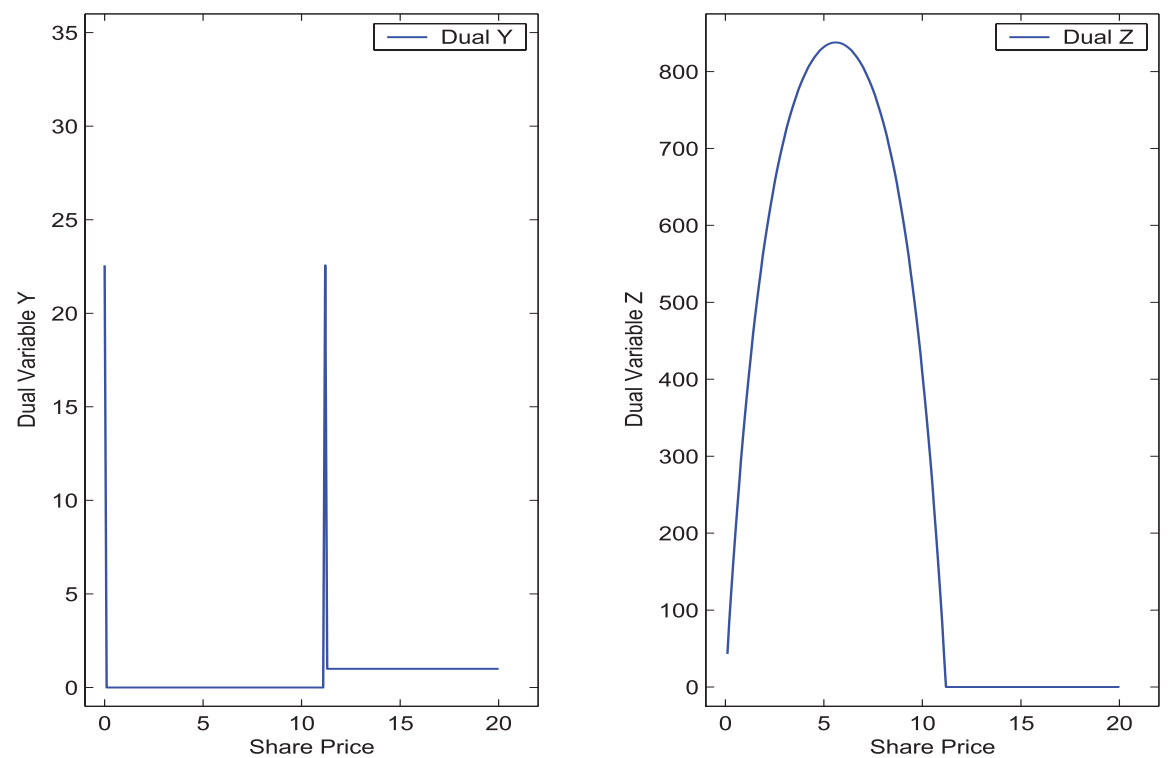

Fig. 4 Plot of optimal values of $y$ and $z$ corresponding to the case where $\alpha=0.999, p=0.5$, $\Delta x=0.1, S=9, j^{*}=112$, and $x_{j^{*}}=11.2$.

The reasoning leading to this property is as follows. If $j$ itself is the index of a local minimum, there is nothing else to prove. Otherwise, there are two possibilities: we have either $z_{j+1}>z_{j}>z_{j-1}$ or $z_{j-1}>z_{j}>z_{j+1}$. In the first case we have either $z_{j-2} \geq z_{j-1}$, in which case $z_{j-1}$ is a local minimum, or $z_{j-2}<z_{j-1}$. If the latter occurs, we can repeat the same argument with the triple $j-3, j-2, j-1$, and we find either $z_{j-2}$ to be a local minimum or $z_{j-3}<z_{j-2}<z_{j-1}$. Continuing in this fashion we are bound to encounter a local minimum either at $z_{1}<0$ (since $z_{0}=0$ ) or earlier. In the second case, the reasoning is identical and yields a local minimum either at $z_{j^{*}-1}<0\left(\right.$ since $\left.z_{j^{*}}=0\right)$ or earlier.

On the other hand, as a consequence of the above observation we also have

$$
\begin{aligned}
z_{k} & =1+\alpha\left(p z_{k-1}+q z_{k+1}\right) \\
& \geq 1+\alpha\left(p z_{k}+q z_{k}\right) \\
& =1+\alpha z_{k} .
\end{aligned}
$$

Rearranging, we get $z_{k} \geq 1 /(1-\alpha)>0$, which contradicts the assumption that $z_{k}$ is negative. Hence, inequalities (8.2) hold for all $j$. This is a simple example of a minimum principle as one encounters in harmonic analysis; see, e.g., [7, 8]. For continuously differentiable functions, the local version of the principle states that any nonconstant harmonic function defined over a domain (in $\mathbb{R}^{n}$ ) not containing $+\infty$ as an interior point cannot attain a local minimum and maximum over the domain in question. Under further technical conditions, according to the global version of the minimum/maximum principle, the largest and the smallest values of a harmonic function on a domain are attained only at the points of the boundary of the domain. For superharmonic functions, which are of interest here since $z$ is an $\alpha$-superharmonic (discrete) function, the following minimum principle is well known. Let $u$ be a superharmonic function in some domain $D$ in $\mathbb{R}^{n}$. If, for any boundary point $\eta \in \partial D$ and 
any $\epsilon>0$, there is a neighborhood $V=V(\eta)$ such that $u(x)>-\epsilon$ in $D \cap V$, then either $u>0$ or $u \equiv 0$ in $D$.

In our simpler, discrete setting the above analysis gave an analogue of the minimum principle for an $\alpha$-superharmonic function $z$ solving (6.4)-(6.6). We have established that the function $z$ is nonnegative for all values of $j$ between 0 and $j^{*}$. Furthermore, it is immediate to check that we cannot have a local minimum value equal to zero for $1<j<j^{*}-1$; i.e., $z_{j}=0$ with $z_{j-1}>0, z_{j+1}>0$ is impossible. It is also obvious that $z$ cannot be identically zero. Therefore, the (global) minimum value is zero and attained only at the two boundary points, i.e., $j=0$ and $j=j^{*}$, which is also observed in the right plot of Figure 4 (recall that $z_{0}=0$ by (6.5)). In the terminology of [6], $z$ is an instance of an excessive function.

8.2. Inequalities (8.I). These follow trivially from inequalities (8.2) and the formula for $y_{j}$.

8.3. Inequalities (8.4). These hold trivially for $j<j^{*}$. They also hold trivially for $j>j^{*}$, provided we assume that $\alpha p \leq 1 / 2$ and $\alpha q \leq 1 / 2$. We need to make an additional assumption! To see this, let $j=j^{*}+k, k=1,2, \ldots$, and assume $\alpha p \leq 1 / 2$ and $\alpha q \leq 1 / 2$. Then

$$
\begin{aligned}
\alpha p v_{j^{*}+k+1}+\alpha q v_{j^{*}+k-1} & =\alpha p f_{j^{*}+k+1}+\alpha q f_{j^{*}+k-1} \\
& \leq \frac{1}{2} f_{j^{*}+k+1}+\frac{1}{2} f_{j^{*}+k-1} \\
& =\frac{1}{2}\left(f_{j^{*}}+(k+1) \Delta x\right)+\frac{1}{2}\left(f_{j^{*}}+(k-1) \Delta x\right) \\
& =f_{j^{*}}+k \Delta x \\
& =f_{j^{*}+k} \\
& =v_{j^{*}+k} .
\end{aligned}
$$

The one just given suffices but is not necessary (the example of Figure 5 shows that the condition is not necessary). A necessary and sufficient condition is obtained by recalling that $v_{j}=f_{j}=x_{j}-S$ for $j>j^{*}$ (even for this we need to check that $x_{j^{*}}>S$ ). It is easy to see after some simple algebraic manipulation that for $j=j^{*}+k$ and $k=1,2, \ldots$, we have

$$
\alpha p v_{j^{*}+k+1}+\alpha q v_{j^{*}+k-1} \leq v_{j^{*}+k}
$$

if and only if

$$
(1-\alpha) f_{j^{*}} \geq \alpha \Delta x(p-q)+k(\alpha-1) \Delta x .
$$

Since $k(\alpha-1) \Delta x$ is negative $(\alpha<1)$, the left-hand side is maximized at $k=1$. Hence, (8.4) holds with $v_{j}=f_{j}$ if and only if

$$
(1-\alpha) f_{j^{*}} \geq \alpha \Delta x(p-q)+(\alpha-1) \Delta x .
$$

Since this condition is somewhat more tedious to check (it requires the computation of $f_{j^{*}}$ ) we use the simpler sufficient conditions $\alpha p \leq 1 / 2, \alpha q \leq 1 / 2$.

We shall come back to inequality (8.4) for $j=j^{*}$ after we consider inequalities (8.3).

Copyright (c) by SIAM. Unauthorized reproduction of this article is prohibited. 
8.4. Inequalities (8.3). For $j \geq j^{*}$, these are trivial. Furthermore, it follows immediately from (7.1) that $v_{j} \geq 0$ for all $j$ (note that the roots $\xi_{+}, \xi_{-}$satisfy $\xi_{-}>1$ and $0<\xi_{+}<1$, provided that $\alpha<1$ ). Hence, we just need to check that $v_{j} \geq x_{j}-S$ for $j<j^{*}$. In order to have these inequalities hold for $j<j^{*}$, we need to pick

$$
j^{*} \in K:=\left\{k: f_{k} \frac{\xi_{+}^{k-1}-\xi_{-}^{k-1}}{\xi_{+}^{k}-\xi_{-}^{k}}>f_{k-1}\right\} .
$$

Of course, we need to assume that $K$ is nonempty. Clearly no $k$ for which $x_{k}<S$ can belong to the set (because both $f_{k}$ and $f_{k-1}$ vanish). For convenience, then, we assume that $S \in E$ - that is, $S=j_{S} \Delta x$ for some $j_{S}$. In that case, we assume that $k=j_{S}+1$ belongs to the set $K$, i.e.,

$$
f_{j_{S}+1} \frac{\xi_{+}^{j_{S}}-\xi_{-}^{j_{S}}}{\xi_{+}^{j_{S}+1}-\xi_{-}^{j_{S}+1}}>f_{j_{S}}
$$

Notice that $K$ is a set with a finite number of elements. To see this, it suffices to observe that for $k \in K$ we have

$$
\frac{\xi_{+}^{k-1}-\xi_{-}^{k-1}}{\xi_{+}^{k}-\xi_{-}^{k-1} \xi_{-}}>\frac{f_{k-1}}{f_{k}}
$$

and hence the left-hand side behaves as $1 / \xi_{-}$and the right-hand side as 1 for large $k$, which implies that $k$ cannot grow without bound since $\xi_{-}>1$. Let $h_{j}=x_{j}-S$. With such a choice and the assumption that $j^{*} \in K$, we have that $v_{j^{*}}=h_{j^{*}}$ and $v_{j^{*}-1}>h_{j^{*}-1}$. Suppose that $v_{j^{\prime}}<h_{j^{\prime}}$ for some $j^{\prime}<j^{*}$. Then the sequence $u_{j}:=$ $v_{j}-h_{j}$ must have a local maximum at some point, say $k$, strictly between $j^{\prime}$ and $j^{*}$. That is, $u_{k}>u_{k-1}$ and $u_{k}>u_{k+1}$. However, we also have

$$
\begin{aligned}
u_{k} & =v_{k}-h_{k} \\
& =\alpha\left(p v_{k+1}+q v_{k-1}\right)-\frac{1}{2}\left(h_{k+1}+h_{k-1}\right) \\
& \leq \frac{1}{2}\left(v_{k+1}+v_{k-1}\right)-\frac{1}{2}\left(h_{k+1}+h_{k-1}\right) \\
& =\frac{1}{2}\left(u_{k+1}+u_{k-1}\right) \\
& <u_{k} .
\end{aligned}
$$

Clearly this is impossible. Hence, $u_{j}$ cannot have a local maximum, and therefore $v_{j}$ cannot dip below $h_{j}$.

8.5. Inequality (8.4) with $\boldsymbol{j}=\boldsymbol{j}^{*}$. Finally, to get inequality (8.4) for $j=j^{*}$, we need to assume that $j^{*}+1 \notin K$. That is,

$$
f_{j^{*}+1} \frac{\xi_{+}^{j^{*}}-\xi_{-}^{j^{*}}}{\xi_{+}^{j^{*}+1}-\xi_{-}^{j^{*}+1}} \leq f_{j^{*}}
$$

To see why, let $w_{j}$ denote the solution to the difference equation

$$
\begin{aligned}
w_{j}-\alpha\left(p w_{j+1}+q w_{j-1}\right) & =0, \quad 0<j, \\
w_{0} & =0 \\
w_{j^{*}} & =f_{j^{*}} .
\end{aligned}
$$

Copyright $@$ by SIAM. Unauthorized reproduction of this article is prohibited. 
This is the same as (6.1)-(6.3) but extended to all $j$. Clearly we have $v_{j^{*}}=w_{j^{*}}$ and $v_{j^{*}-1}=w_{j^{*}-1}$. Hence, (8.4) at $j^{*}$ will hold if and only if $v_{j^{*}+1} \leq w_{j^{*}+1}$ :

$$
f_{j^{*}+1}=v_{j^{*}+1} \leq w_{j^{*}+1}=f_{j^{*}} \frac{\xi_{+}^{j^{*}+1}-\xi_{-}^{j^{*}+1}}{\xi_{+}^{j^{*}}-\xi_{-}^{j^{*}}} .
$$

The resulting inequality is clearly equivalent to (8.6).

A natural consequence of our analysis above is that $j^{*}$ should be chosen as

$$
j^{*}:=\max \left\{k: f_{k} \frac{\xi_{+}^{k-1}-\xi_{-}^{k-1}}{\xi_{+}^{k}-\xi_{-}^{k}}>f_{k-1}\right\} .
$$

9. Summary. The claimed result holds, provided we make the assumptions that

1. $\alpha p \leq 1 / 2$ and $\alpha q \leq 1 / 2$,

2. $S=j_{S} \Delta x$ for some integer $j_{S}$, and

3. $f_{j_{S}+1}\left(\xi_{+}^{j_{S}}-\xi_{-}^{j_{S}}\right) /\left(\xi_{+}^{j_{S}+1}-\xi_{-}^{j_{S}+1}\right)>f_{j_{S}}$.

With these assumptions, the optimal choice of $j^{*}$ is

$$
j^{*}:=\max \left\{k: f_{k} \frac{\xi_{+}^{k-1}-\xi_{-}^{k-1}}{\xi_{+}^{k}-\xi_{-}^{k}}>f_{k-1}\right\} .
$$

The result of the present paper holds even if the assumption $\alpha p \leq 1 / 2$ is violated. Such a case is illustrated in Figure 5, where $\alpha p>1 / 2$. However, in this case, the

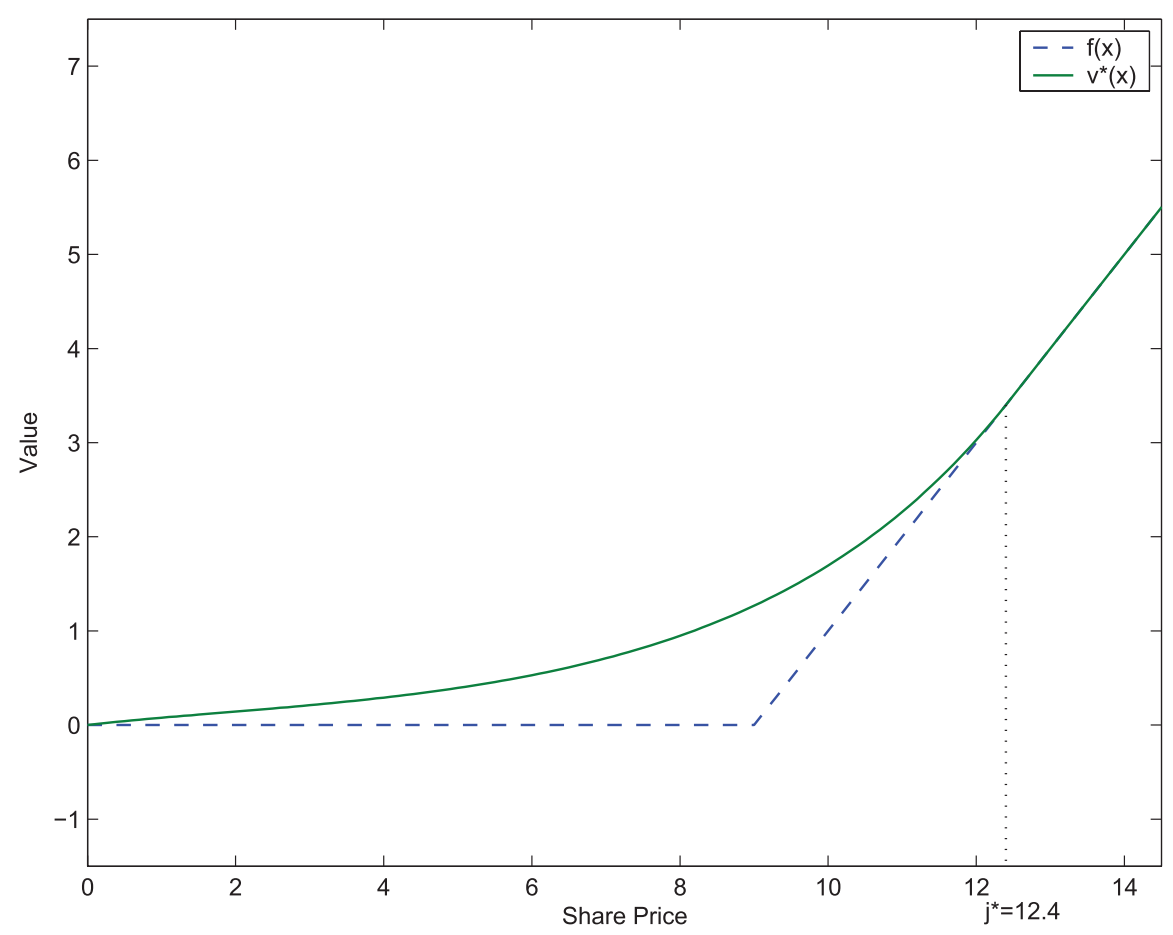

Fig. 5 Plot of $v^{*}(x)$ and $f(x)$ corresponding to the case where $\alpha=0.999, p=0.51, \Delta x=0.1$, and $S=9$. In this case, $j^{*}=124$ and therefore $x_{j^{*}}=12.4$.

Copyright $@$ by SIAM. Unauthorized reproduction of this article is prohibited. 
necessary and sufficient condition (8.5) holds. Low interest rates (i.e., $\alpha$ values very close to one) and an upward-biased $(p>1 / 2)$ stock movement model may easily induce a violation of the assumed upper bound of $1 / 2$ on $\alpha p$. Such cases certainly make sense economically. The more involved necessary and sufficient condition (8.5) can be checked for these cases, provided that assumptions 2 and 3 of section 1 hold.

We conclude the paper with two exercise suggestions. The analysis and results presented here remain valid if, for the stock price,

(a) we use a Markov chain model with absorption at zero, where at each step the stock price moves up by $\Delta x$ with probability $p$, and moves down by $\Delta x$ with probability $q$ (with $p+q<1$ ), and does not change with probability $1-(p+q)$ (this is a model closer to reality for the movement of stock prices over a short time interval); and

(b) we use a geometric random walk model where the stock price at the next period becomes $x \lambda$ with probability $p$, and $x / \lambda$ with probability $1-p$, where $\lambda>1$ and the current price is $x$.

We provide some guidelines and hints below on the extension of our analysis to these two cases.

For case (a), the analysis and results hold with a slight modification in the valuation formula. Adjusting the second set of constraints in our original linear programming model, and rearranging the terms of this constraint, one can see that an almost identical model solves the valuation problem at hand, and only the right-hand side of the second constraint set is subject to change. This feature leads to a change in the structure of the difference equations in our original problem, which in turn leads to a slight change in the roots of the difference equation. The formulae for $v, y$, and $z$ retain the same form except for a slight change in the specified roots.

Case (b) is slightly more involved in that the state space is constructed from some initial price, and consequently one deals with the state space $E^{m}=\left\{X_{0}^{m} \lambda^{j}: j \in \mathbb{Z}\right\}$, where $X_{0}^{m}$ is the initial stock price. The value function should now tend to zero when $j \rightarrow-\infty$, whereas in the problem treated in the previous sections we had $v(0)=0$. The key to the solution lies in using this modified boundary condition to obtain a particular solution to the second-order difference equations of section 7 .

Acknowledgment. The second author gratefully acknowledges the assistance of Efe B. Bozkaya during the revision.

\section{REFERENCES}

[1] R. E. Bellman and S. E. Dreyfus, Applied Dynamic Programming, Princeton University Press, Princeton, NJ, 1962.

[2] S. Boyd and L. Vandenberghe, Convex Optimization, Cambridge University Press, Cambridge, UK, 2004.

[3] A. H. Y. Chen, A Model of Warrant Pricing in a Dynamic Market, J. Finance, 25 (1970), pp. 1041-1059.

[4] E. ÇInlaR, Introduction to Stochastic Processes, Prentice-Hall, Englewood Cliffs, NJ, 1975.

[5] J. Detemple, American-Style Derivatives: Valuation and Computation, Chapman Hall/CRC Financ. Math. Ser., Chapman \& Hall/CRC, Boca Raton, FL, 2006.

[6] E. B. Dynkin And A. A. Yushkevich, Markov Processes: Theorems and Problems, Plenum Press, New York, 1969; translated from the Russian by James S. Wood.

[7] D. Gilbarg and N. S. Trudinger, Elliptic Partial Differential Equations of Second Order, Grundlehren Math. Wiss. 224, Springer, Berlin, 1977.

[8] M. Hazewinkel, ED., The Online Encyclopaedia of Mathematics, Springer, http://eom. springer.de/default.htm. 
[9] J. D. Hoffmann, Numerical Methods for Engineers and Scientists, 2nd ed., Marcel Dekker, New York, 2001.

[10] E. P. C. KaO, An Introduction to Stochastic Processes, Duxbury Press, Belmont, CA, 1997.

[11] S. Karlin and H. M. Taylor, A First Course in Stochastic Processes, 2nd ed., Academic Press, New York, 1975.

[12] P. A. Samuelson, Rational Theory of Warrant Pricing, Indust. Management Rev., 6 (1965), pp. 13-31; appendix by H. P. McKean, pp. 32-39.

[13] L. SHEPP, A Model for Stock Price Fluctuations Based on Information, IEEE Trans. Inform. Theory, 48 (2002), pp. 1372-1378.

[14] R. J. VAnderbei, Linear Programming: Foundations and Extensions, 2nd ed., Springer, New York, 2001.

Copyright (c) by SIAM. Unauthorized reproduction of this article is prohibited. 